\title{
CRISPR/CAS9 as a Powerful Tool for Crop Improvement
}

\author{
Jae-Young Song $\cdot$ Marjohn Niño $\cdot$ Franz Marielle Nogoy $\cdot$ Yu-Jin Jung $\cdot$ Kwon-Kyoo Kang $\cdot$ Yong-Gu Cho
}

Received: 4 June 2017 / Revised: 5 June 2017 / Accepted: 21 June 2017

(C) Korean Society for Plant Biotechnology

\begin{abstract}
Implementation of crop improvement programs relies on genetic diversity. To overcome the limited occurrence of natural mutations, researchers and breeders applied diverse methods, ranging from conventional crossing to classical biotechnologies. Earlier generations of knockout and gain-offunction technologies often result in incomplete gene disruption or random insertions of transgenes into plant genomes. The newly developed editing tool, CRISPR/Cas9 system, not only provides a powerful platform to efficiently modify target traits, but also broadens the scope and prospects of genome editing. Customized Cas9/guide RNA (gRNA) systems suitable for efficient genomic modification of mammalian cells or plants have been reported. Following successful demonstration of this technology in mammalian cells, CRISPR/Cas9 was successfully adapted in plants, and accumulating evidence of its feasibility has been reported in model plants and major crops. Recently, a modified version of CRISPR/Cas9 with added novel functions has been developed that enables programmable direct irreversible conversion of a target DNA base. In this review, we summarized the milestone applications of CRISPR/ Cas9 in plants with a focus on major crops. We also present the implications of an improved version of this technology in the current plant breeding programs.
\end{abstract}

Keywords Base targeting, CRISPR/Cas9, Genome editing, Crop improvement

\footnotetext{
${ }^{\dagger}$ First two authors equally contributed.

J.-Y. Song ${ }^{\dagger} \cdot$ M. Niño ${ }^{\dagger} \cdot$ F. M. Nogoy • Y.-G. Cho $(\bowtie)$

Department of Crop Science, Chungbuk National University, Cheongju 28644, Korea

e-mail: ygcho@cbnu.ac.kr

Y.-J. Jung $\cdot$ K.-K. Kang

Department of Horticulture, Hankyong National University, Anseong 17579, Korea
}

\section{Introduction}

Recent advances in genome engineering technologies offer tremendous opportunities to accelerate crop functional genomics studies in a bid to provide robust data for crop improvement. Application of nucleases as editing tool has come a long way in modifying genomes not only with model organisms but even those with genetically challenging components. To date, CRISPR/Cas9 system, has emerged as the most effective genome editing tool overcoming the limitations faced by earlier technologies such as the limited choice of targets with ZFN due to context-dependent effects between individual finger domains in an array (Wolfe et al. 2000; Sanders and Joung 2014) and the delivery issues with TALEN for certain viral vectors (Holkers et al. 2013; Sanders and Joung 2014). Unlike the predecessor zinc finger nuclease (ZFN) and TAL effector nuclease (TALEN), which involve dimerizing fusion proteins including the DNA binding domains of ZF and TAL and cleavage domains of FokI endonuclease, Cas9/gRNA is a ribonucleoprotein active on target DNA (Char et al. 2017). This technology has been widely adopted to study important genes in the cell of mice (Mashiko et al. 2014), monkeys (Niu et al. 2014), and other organisms, including bacteria (Fabre et al. 2014), yeast (DiCarlo et al. 2013), zebrafish (Hwang et al. 2013), Drosophila (Gratz et al. 2014), rabbits (Yang et al. 2014), pigs (Hai et al. 2014), rats (Ma et al. 2014), human (Mali et al. 2013), and plants (Mali et al. 2013). In biomedical field, significant applications have already been achieved including correcting human genetic disorder, treatment of the acquired immune deficiency syndrome (AIDS) or promoting anti-tumor immunotherapy, and genetic manipulation of domesticated animals for production of biologic medical materials among others (Cai et al. 2016). While CRISPR/Cas9 application is expected to fast track molecular breeding without retention of transgene components in the plant product, it also faces a number of hurdles obstracting its maximum potential use especially 
in crops with large, polyploid genomes (Cram et al. 2017). This was dealt by developing powerful complementary bioinformatics tools that would facilitate full implementation of CRISPR/Cas9 technology in plant genetic engineering. Nevertheless, this technology has been successfully applied in several plant systems, in fact quite a number of reports have been published since its discovery. As this technique rapidly evolves, its application is constantly expanding which can be adapted to spur plant breeding for certain traits that are too challenging using conventional techniques.

Principle of CRISPR/Cas9 and creating variation

Genome editing technologies have been developed to induce site-specific DNA cleavage. The site-specific nucleases (SSNs) can induce double-strand break (DSB) specifically in targeted loci of genomic DNA for genetic improvement in any organisms (Jinek et al. 2012; Cong et al. 2013; Mali et al. 2013). The SSNs system can be classified mainly into three classes, which are zinc finger nucleases (ZFNs) (Kim et al. 1996), transcriptional activator-like effector nucleases (TALENs) (Christian et al. 2010), and the CRISPR/ Cas9 nuclease system from Streptococcus pyogenes (Jinek et al. 2012). Among them, the CRISPR/Cas9 system is originated from the immune response system of bacteria which protect themselves by cleaving the DNA of invading viruses (Bortesi and Fischer 2015). To create variation by gene cleavages, Cas9 nuclease and single guide RNA (sgRNA) complex recognizes the protospacer-adjacent motif (PAM), which is $5^{\prime}$-NGG-3' in the target site and binds a specific genomic location guided by a short sequence ( $20 \mathrm{bp})$ in sgRNA, and then Cas9 cleaves target DNA at 3 bp upstream of the PAM (Jinek et al. 2012). The PAM sequence plays an important role in binding and breaking to the target DNA (Sternberg et al. 2014). The CRISPR/Cas system repairs targeted DNA location very specifically by either via the non-homologous end joining (NHEJ) DNA repair pathway (Rouet et al. 1994) or the homology directed repair (HDR) (Bibikova et al. 2002) pathway with cellular DNA repair mechanisms (Abdallah et al. 2015; Luo et al. 2017). DSB repair through the NHEJ can generate gene knock-outs via small insertions or deletions conferring loss-of-function at the repair positions, while HDR can lead to gene knock-ins as precise sequence alterations for gene replacement conferring gain-of-function (Altpeter et al. 2017; Puchta and Fauser 2014; Voytas and Gao 2014) (Fig. 1). In plants, most of the reported gene editing events was mediated by NHEJ repair mechanism to generate mutations and gene knock-outs. However, gene replacement rather than gene inactivation will greatly facilitate and improve plant breeding by allowing the introduction of precise point mutations or enabling new functions. HDR-based repair generated by CRISPR/Cas9 potentially can provide a feasible approach to achieve gene replacement but currently suffers from very low efficiencies (Lowder et al. 2016; Sun 2017).

\section{Milestones of CRISPR/Cas9 in crop biotechnology}

Different Cas9/gRNA systems have been tailored for effective genomic alterations in both prokaryotes and eukaryotes (Char et al. 2017). Following successful demonstration in mammalian cells, this technology has been adapted in some plant species, and accumulating evidences of its feasibility have been reported in rice (Feng et al. 2013; Jiang et al. 2013; Shan et al. 2013; Zhang et al. 2014; Wang et al. 2016), maize (Feng et al. 2015; Svitashev et al. 2015; Zhu et al. 2015; Char et al. 2017), wheat (Shan et al. 2013; Wang et al. 2014), tomato (Brooks et al. 2014; Cermak et al. 2015), soybean (Li et al. 2015), and potato (Wang et al. 2015). Rice, being a diploid and a monocot plant, has been one of the top choices among researchers for CRISPR/Cas9 demonstration. Some of the pioneering works done in rice involved targeting the promoter regions of bacterial blight susceptibility genes, OSSWEET14 and OsSWEET11 (Jiang et al. 2013). In this study, precise editing was mediated by two constructs including $S$. pyogenes Cas9 enzyme expressed under CaMV 35S and a chimeric sgRNA driven by a rice U6 gene promoter, which contains a 5 ' region complementary to a sequence of $20 \mathrm{bp}$ in the promoter of OSSWEET14 and a 3' sgRNA scaffold that recruits Cas9. These constructs were successfully transformed into rice using PEG-protoplast transfection protocol. The use of this delivery method clearly showed that mutagenesis by Cas9/sgRNA complex indeed occurs within the plant cells which are free of any bacterial cell involvement (Jiang et al. 2013). The group also demonstrated that codon optimization of the Cas9 positively influenced mutagenesis rate, in this case, using the other bacterial blight susceptibility gene, OsSWEET11. Using the same technique down to codon optimization of the Cas9, Shan et al. (2013) targeted four rice genes, phytoene desaturase $(O S P D S)$, betaine aldehyde dehydrogenase $(\mathrm{OSBADH2})$, basic helix-loop-helix (bHLH) transcription factor $(O s O 2 g 23823)$, and mitogen-activated protein kinase $\left(O_{s M P K 2}\right)$, in separate constructs where sgRNA was expressed by U3 promoter. Each of the endogenous genes was specifically disrupted using only single sgRNA, except for $O S P D S$, which was targeted by two sgRNAs. On average, high mutagenesis frequency rates were observed 
A

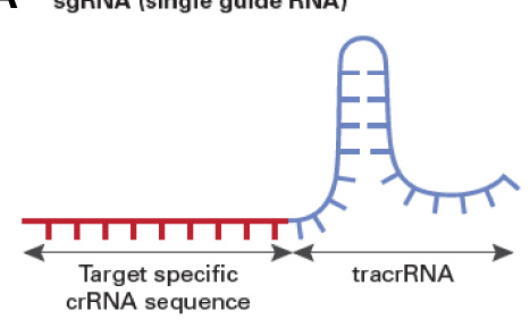

C Target specific cleavage

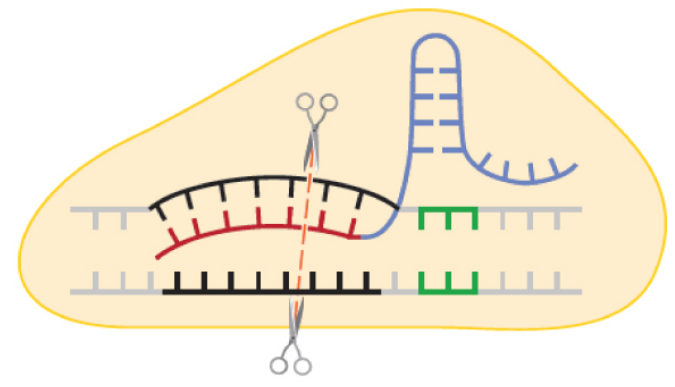

E Breeders can selecta null segregant by selfing

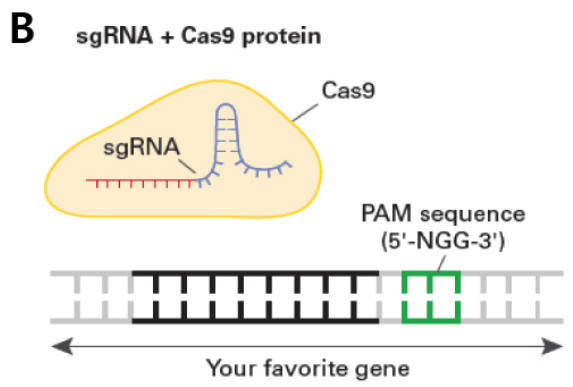

D Cellular error-prone repair "knocks out" gene

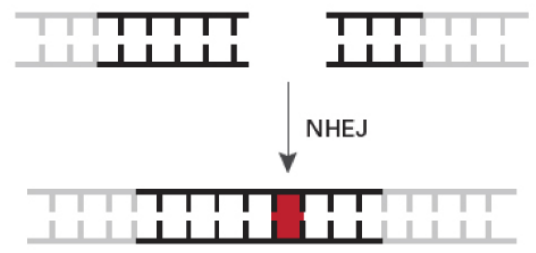

*http://www.clontech.com/US/Products/Genome_Editing/CRISPR_Cas9 Resources/About_CRISPR Cas9

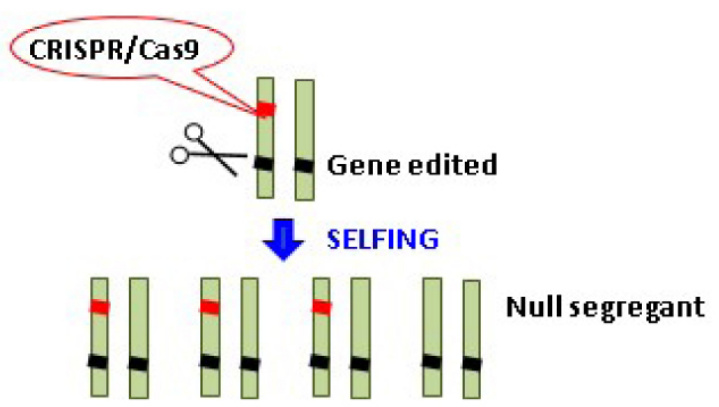

Fig. 1 Process showing CRISPR/Cas9 system as a powerful tool for crop improvement. (A) Designing single guide RNA (sgRNA), (B) Engineering CRISPR/Cas9 nucleases with altered protospacer adjacent motif (PAM), (C) Targeting specific cleavage in plant genome, (D) Editing gene by non-homologous end joining (NHEJ) with CRISPR/Cas9, (E) Selecting null segregants in the next generation

in both rice protoplast cells and transgenic plants (Table 1). It has been reported that \% GC content, targeting strand and targeting context of sgRNA targeting sequences may influence sgRNA efficacy (Wang et al. 2014). This was successfully demonstrated by Zhang et al. (2014), wherein all, except one, of the 11 sgRNAs examined, showed higher editing efficiency. This group investigated the amenability of 11 rice genes to CRISPR/Cas9 system designed to be disrupted singly and in combination using Agrobacteriummediated delivery (Table 1). It's commonly known that homozygous or bi-allelic $\mathrm{T}_{0}$ plants occur when both copies of the target gene in rice are mutated before the first division of the embryogenic cell. In the study conducted by Zhang et al. (2014), $7.7 \%$ of the plants carrying targeted mutations were found to contain homozygous mutations, which implies that CRISPR/Cas9 acts early during the regeneration of $\mathrm{T}_{0}$ plants. Further, simultaneous targeting of two genes for four pairs of targets including OsMSH1 and OsDERF1, OsMSH1 and $O S P D S, O S P D S$ and $O S P M S 3$, and $O S P D S$ and $O S D E R F 1$ yields high site-specific mutation rates (Table 2) which are not too different from the expected double mutation rates, that is, the mutation rate at one sites times the rate at the other site. These suggest that mutations at the two sites targeted by one construct occurred independently of each other (Zhang et al., 2014). Application of this technology in dicot plants including tomato (Brooks et al. 2014; Cermak et al. 2015), Soybean ( $\mathrm{Li}$ et al. 2015), and potato (Wang et al. 2015) have also yielded high site-specific target mutations, thus pointing out the feasibility of CRISPR/Cas9 in most, if not all, plant systems indicating its potential to revolutionize the genome editing of economically important crops. 
Table 1 Single gene editing by CRISPR/Cas9 in major crops

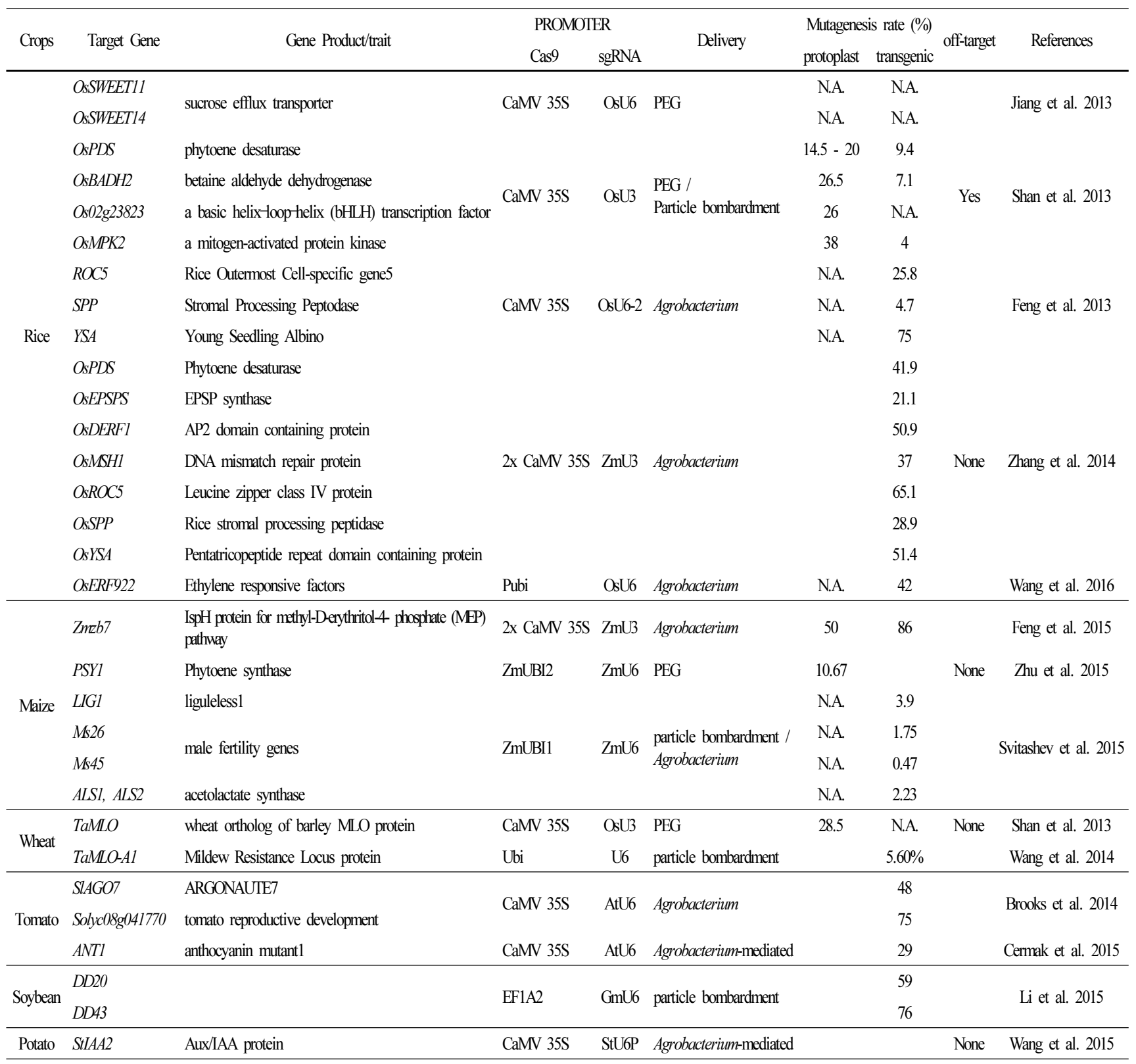

Table 2 Double gene editing by CRISPR/Cas9 in selected major crops

\begin{tabular}{|c|c|c|c|c|c|c|c|c|c|}
\hline \multirow{2}{*}{ Crops } & \multirow{2}{*}{ Gene } & \multirow{2}{*}{ Gene product/Trait } & \multirow{2}{*}{ Delivery } & \multicolumn{2}{|c|}{ PROMOTER } & \multicolumn{3}{|c|}{ Mutagenesis frequency (\%) } & \multirow{2}{*}{ References } \\
\hline & & & & Cas9 & sgRNA & Target 1 & Target 2 & Both & \\
\hline \multirow[t]{3}{*}{ Maize } & $L I G, M S 26$ & \multirow{3}{*}{$\begin{array}{l}L I G \text { (liguleless) } \\
M S 26 \& 45 \text { (male sterility) }\end{array}$} & \multirow{3}{*}{$\begin{array}{c}\text { particle bombardment / } \\
\text { Agrobacterium }\end{array}$} & \multirow{3}{*}{ ZmUbi } & \multirow{3}{*}{ ZmU6 } & \multicolumn{3}{|r|}{$1.43 \%-1.78 \%$} & \multirow{3}{*}{$\begin{array}{l}\text { Svitashev } \\
\text { et al. } 2015\end{array}$} \\
\hline & MS26, MS45 & & & & & & & $1.62 \%-1.67 \%$ & \\
\hline & LIG, MS26, MS45 & & & & & & & $1.55 \%-1.86 \%$ & \\
\hline \multirow{4}{*}{ Rice } & OsMSH1, OsDERF1 & \multirow{4}{*}{$\begin{array}{l}\text { OsMSH1(pleiotrophic phenotype); } \\
\text { OsDERF1(drought-resistant); } \\
\text { OsPDS(albino); } \\
\text { OsPMS3(non-coding RNA) }\end{array}$} & \multirow{4}{*}{ Agrobacterium } & \multirow{4}{*}{ 2x CaMV 35S } & \multirow{4}{*}{$\mathrm{ZmU} 3$} & 50 & 56.7 & 33.3 & \multirow{4}{*}{$\begin{array}{l}\text { Zhang } \\
\text { et al. } 2014\end{array}$} \\
\hline & OsMSH1, OsPDS & & & & & 40 & 56.4 & 32.7 & \\
\hline & OsPDS, OsPMS3 & & & & & 31.4 & 17.1 & 8.6 & \\
\hline & OsPDS, OsDERF1 & & & & & 37.1 & 17.1 & 5.7 & \\
\hline Tomato & $\begin{array}{l}\text { Solyc } 07 g 021170, \\
\text { Solyc } 12 g 044760\end{array}$ & tomato reproductive development & Agrobacterium & CaMV 35S & AtU6 & & & 100 & $\begin{array}{l}\text { Brooks } \\
\text { et al. } 2014\end{array}$ \\
\hline
\end{tabular}


Up until now, most of the reported gene editing events in plants is mediated by an error-prone NHEJ repair mechanism, and only limited evidence that HDR-mediated gene insertion works well in plants have been presented so far due to impractically low frequencies. The Cas9-gRNA system was tested for its ability to facilitate targeted gene insertion in maize immature embryo cells (Svitashev et al. 2015). Here, the DNA donor repair template contained the constitutively expressed PAT gene (UBI:MoPAT) flanked by approximately $1.0 \mathrm{~K}$ of DNA fragments homologous to genomic sequences immediately adjacent to the Liguless1 (LIG) cleavage site. Interestingly, only particle bombardment yielded target site-specific gene insertion, and that, donor template DNA designed on the same plasmid together with Cas9-sgRNA resulted in doubled high integration event rate compared when components were delivered as separate vectors. However, this does not necessarily mean that HDR does not work through Agrobacterium-mediated delivery but more likely indicates lower frequency of integration events (Svitashev et al. 2015).

Target base editing using modified CRISPR/Cas9 system

Creation of a much more powerful CRISPR tool box has been reported very recently. The new technique called "base editing" was first demonstrated in mammalian cells, which enables direct and irreversible conversion of one target base into another in a programmable manner, without requiring dsDNA DSB or a donor template (Komor et al. 2016). This new system utilized catalytically modified Cas9 (dCas9) fused to a cytidine deaminase enzyme encoded by the rat APOBEC1 gene. dCas9, which possessed Asp10Ala and His840Ala mutations inactivating its nuclease activity, retains its DNA binding ability via guide RNA, but does not cleave the DNA backbone (Jinek et al. 2012). Instead, this cytidine deaminase converts cytosine $(\mathrm{C})$ bases into uridines (U) (Kuscu and Adli 2016), thereby effecting a $15 \%-75 \%$ of $\mathrm{C} \rightarrow \mathrm{T}$ (or $\mathrm{G} \rightarrow \mathrm{A}$ ) substitution in human cells (Komor et al. 2016), which are then repaired by error-prone mechanisms that result in various point mutations. Fusion of rat APOBEC1 to the amino terminus, but not that carboxy terminus of dCas9, is responsible for a preserved deaminase activity (Komor et al. 2016). Early demonstration in human cells by Komor et al. (2016) yielded no detectable base editing at the known dCas9 off-target sites, and that, base editors in human cells do not induce untargeted $\mathrm{C} \rightarrow \mathrm{T}$ conversion throughout the genome. Later on, $\mathrm{Lu}$ and $\mathrm{Zhu}$ (2016) tested the applicability of this modified CRISPR/Cas9 system in the rice callus of Zhonghua11 (ZH11) using Agro- bacterium-mediated transformation. In their study, they fused rat APOBEC1 to the N-terminus of Cas9(D10A) using the unstructured 16-residue peptide XTEN as linker, and constructed it into a binary vector containing maize ubiquitin promoter. This system was used to edit two agriculturally important genes in rice, $N R T 1.1 B$ and SLR1. Previous reports claimed that a $\mathrm{C} \rightarrow \mathrm{T}$ variation (Thr327Met) in NRT1.1B could increase nitrogen use efficiency in rice $(\mathrm{Hu}$ et al. 2015), while the amino acid substitution in or near TVHYNP of the DELLA protein encoded by SLRI could reduce plant height (Ikeda et al. 2001; Asano et al. 2009). Results showed similar base-editing results in human cells, wherein respective $\mathrm{C} \rightarrow \mathrm{T}$ substitution frequency in $N R T 1.1 B$ and SLRI was $1.4 \%-11.5 \%$, while $\mathrm{C} \rightarrow \mathrm{G}$ replacement frequency was at $1.6 \%-3.9 \%$. Lu and Zhu (2016) declared that the possible cause of the lower base-editing efficiency on $N R T 1.1 B$ was the lower targeting efficiency of the gRNA for this gene. Furthermore, to demonstrate feasibility of this new approach in plant breeding program, $\mathrm{Lu}$ and $\mathrm{Zhu}$ (2016) generated stable transgenic seedlings from the hygromacin-resistant callus. Sanger sequencing and RFLP results of SLR1 consistently confirmed the base replacement in the six transgenic lines which showed a semi-dwarf phenotype. The compelling evidence shown by these studies put CRISPR base-editing technology at the forefront of advancing the efficiency and scope of genome editing.

Implications of improved genome editing in current plant breeding programs

Current challenges in agriculture can be summed up to how we can improve crop production in harsh climatic conditions. Abiotic stress such as drought, salinity, heat, cold, flooding, and radiation are major threat in securing high yield especially of major crops across the globe. To cope these challenges, plants resort to induction of complex interactions among various components of several signaling, regulatory and metabolic pathways (Nakashima et al. 2009), thereby involving multiple genes. For the past decades, generation of stresstolerant crops have been demonstrated using conventional breeding or classical biotechnologies. However, aside from time-constraint, lack of precision proved to be the bottleneck of these classical approaches. Research works for past five years presented compelling evidence that multiplex genome editing is feasible using CRISPR/Cas9 not only in model plants but also in major crops. This provides opportunities to understand the complexity of major traits in crops by deciphering the functions of multiple genes involved in a single trait. Another approach could be the pyramiding of 
multiple genes involved in a stress response pathway or regulatory network via HDR-mediated gene targeting (Jain 2015). Most known agriculturally important traits, however are conferred by point mutations (Huang et al. 2010). Hence, techniques that enable precise and efficient base replacement in the target locus, rather than stochastic disruption of the gene, will greatly facilitate precision plant molecular breeding (Lu et al. 2016). The development of a new approach, base editing, conferred novel functionality that is more powerful than the former CRISPR/Cas9 version, wherein it can directly convert irreversibly one target DNA base into another without DSB or a donor template (Komor et al. 2016). This platform can be harnessed to induce genetic variation which is a key component for crop improvement programs.

These advances in genome editing also allow breeders to select null segregant lines, which lack the CRISPR/Cas9 component, by selfing (Fig. 1). The resulting product is expected to be identical to the classically bred plants (Giddings et al. 2012; Khatodia et al. 2016).

\section{Acknowledgments}

This research was supported by a grant from the NextGeneration BioGreen 21 Program (National Center for GM Crops No. PJ01191601), Rural Development Administration, and from the National Research Foundation (NRF) programs (2014R1A2A1A11052547) funded by the Korean Ministry of Science, ICT and Future Planning, Republic of Korea.

\section{References}

Abdallah NA, Prakash CS, McHughen AG. (2015) Genome editing for crop improvement: Challenges and opportunities. GM Crops \& Food. 6:183-205

Altpeter F, Kannan B, Jung JH, Oz TM, Karan R, Merotto A. (2017) Genetic Improvement of Sugarcane by Targeted Lossor Gain of Function Mutations using TALEN or CRISPR-Cas9. Proc. of the Plant and Animal Genome Conference (PAG XXV). San Diego, CA, USA. W699

Asano K, Hirano K, Ueguchi-Tanaka M, Angeles-Shim RB, Komura T, Satoh H, Kitano H, Matsuoka M, Ashikari M. (2009) Isolation and characterization of dominant dwarf mutants, Slr1-d, in rice. Mol. Genet. Genomics 281:223-231

Bibikova M, Golic M, Golic KG, Carroll D. (2002) Targeted chromosomal cleavage and mutagenesis in Drosophila using zinc-finger nucleases. Genetics 161:1169-1175

Bortesi L, Fischer R. (2015) The CRISPR/Cas9 system for plant genome editing and beyond. Biotechnology Advances 33:41-52

Brooks C, Nekrasov V, Lippman ZB, Eck JV. (2014) Efficient gene editing in tomato in the first generation using the clustered regularly insterspaced short palindromic repeats/ CRISPR-Associated9 system. Plant Physiology. 166:1292-1297

Cai L, Fisher AL, Hunag H, Xie Z. (2016) CRISPR-mediated genome editing and human diseases. Genes \& Diseases 3: 244-251

Čermák T, Baltes NJ, Čegan R, Zhang Y, Voytas DF. (2015) High-frequency. Precise modification of the tomato genome. Genome Biology DOI 10.1186/s13059-015-0796-9

Char SN, Neelakandan AK, Nahamoun H, Frame B, Main M, Soalding MH, Becraft PW, Meyers BC, Walbot V, Wang K, Yang B. (2017) An Agrobacterium-delivered CRISPR/Cas9 system for high frequency targeted mutagenesis in maize. Plant Biotechnology Journal 15: 257-268 doi: 10.1111/pbi.12611

Cong L, Ran FA, Cox D, Lin S, Barretto R, Habib N, Hsu PD, Wu X, Jiang W, Marraffini LA, Zhang F. (2013) Multiplex genome engineering using CRISPR/Cas systems. Science 339:819-823

Cram D, Kulkarni M, Rozwadowski K, Sharpe AG, Kagale S. (2017) Wheat CRISPR: A Web-Based Optimized sgRNA Designer for CRISPR-Cas9-Mediated Genome Editing in Wheat. Proc. of the Plant and Animal Genome Conference (PAG XXV). San Diego, CA, USA. P0821

DiCarlo JE, Norville JE, Mali P, Rios X, Aach J, Church GM. (2013) Genome engineering in Saccharomyces cerevisiae using CRISPR-Cas systems. 41(7):4336-43. doi: 10.1093/nar/gkt135

Fabre L, Le Hello S, Roux C, Jeanjean SI, Weill FX. (2014) CRISPR Is an Optimal Target for the Design of Specific PCR Assays for Salmonella enterica Serotypes Typhi and Paratyphi A. PLoS Negl Trop Dis. 8(1): e2671. 10.1371/journal.pntd. 0002671

Feng C, Yuan J, Wang R, Liu Y, Birchler JA, Han F. (2015) Efficient targeted genome modification in maize using CRISPR/Cas9 system. Journal of Genetics and Genomics 43: 37-43

Feng Z, Zhang B, Ding W, Liu X, Yang DL, Wei P, Cao F, Zhu S, Zhang F, Mao Y, Zhu JK. (2013) Efficient genome editing in plants using CRISPR/Cas system. Cell Research 23:12291232 doi:10.1038/cr.2013.114

Giddings LV, Potrykus I, Ammann K, Fedoroff NV. (2012) Confronting the Gordian knot.

Nat.Biotechnol. 30, 208-209.doi:10.1038/nbt.2145

Gratz SJ, Ukken FP, Rubinstein CD, Thiede G, Donohue LK, Cummings AM, O'Connor-Giles KM. (2014) Highly specific and efficient CRISPR/Cas9-catalyzed homology-directed repair in Drosophila. Genetics 196(4):961-71. doi: 10.1534/ genetics. 113.160713

Hai T, Teng F, Guo R, Li W, Zhou Q. (2014) One-step generation of knockout pigs by zygote injection of CRISPR/Cas system Cell Res. 24(3): 372-375 doi: 10.1038/cr.2014.11

Huang X, Wei X, Sang T, Zhao Q, Feng Q, Zhao Y, Li C, Zhu C, Lu T, Zhang Z,Li M, Fan D, Guo Y, Wang A, Wang L, Deng L, Li W, Lu Y, Weng Q, Liu K, Huang T, Zhou T, Jing Y, Li W, Lin Z, Bucker ES, Qian Q, Zhang QF, Li J, Han B. (2010) Genome-wide association studies of 14 agronomic traits in rice landraces. Nat. Genet. 42:961-967

Hwang WY, Fu Yanfang, Reyon Deepak, Maeder ML, Kaini P, 
Sander JD, Joung K, Peterson RT, Yeh JYJ. (2013) Heritable and Precise Zebrafish Genome Editing Using a CRISPR-Cas System. PLOS One 8:e68708. doi.org/10.1371/journal.pone. 0068708

Holkers M, Maggio I, Liu J, Janssen JM, Miselli F, Mussolino C, Recchia A of TALE nuclease genes, Cathomen T, Goncalves MA. (2013) Differently integrity of TALE nuclease genes following adenoviral and lentiviral vector gene transfer into human cells. Nucliec Acids Res. 1;41(5):e63. doi: 10.1093/ nar/gks1446

Hu B, Wang W, Ou S, Tang J, Li H, Che R, Zhang Z, Chai X, Wang H, Wang Y, Liang C, Liu L, Piao Z, Deng Q, Deng K, Xu C, Liang Y, Zhang L, Li L, Chu C. (2015) Variation in NRT1.1B contributes to nitrate-use divergence between rice subspecies. Nat. Genet. 47:834-838

Ikeda A, Ueguchi-Tanaka M, Sonoda Y, Kitano H, Koshioka M, Futsuhara Y, Matsuoka M, Yamaguchi J. (2001) Slender rice, a constitutive gibberellin response mutant, is caused by a null mutation of the SLR1 gene, an ortholog of the heightregulating gene GAI/RGA/RHT/D8. Plant Cell 13:999-1010

Jain M. (2015) Function genomics of abiotic stress tolerance in plants: a CRISPR approach. Front. Plant Sci. 6:375. Doi: 10.3389/fpls.20115.00375

Jiang W, Bikard D, Cox D, Zhang F, Marraffini LA. (2013) RNA-guided editing of bacterial genomes using CRISPR-Cas systems. Nat Biotechnol 31: 233-239

Jinek M, Chylinski K, Fonfara I, Hauer M, Doudna JA, Charpentier E. (2012) A programmable dual-RNA-guided DNA endonuclease in adaptive bacterial immunity. Science 337:816-21

Khatodia S, Bhatotia K, Passricha N, Khurana SMP, Tuteja N. (2016) The CRISPR/Cas Genome-EditingTool: Application in Improment of Crops. Frontiers in Plant Science 7:506.doi: 10.3389/fpls.2016.00506

Kim YG, Cha J, Chandrasegaran S. (1996) Hybrid restriction enzymes: zinc finger fusions to Fok I cleavage domain. Proc Natl Acad Sci USA 93:1156-60

Komor AC, Kim YB, Packer MS, Zuris JA, Liu DR. (2016) Programmable editing of a target base in genomic DNA without double-stranded DNA cleavage. Nature 533:420-426

Kuscu C, Arslan S, Singh R, Thorpe J, Adli M. (2014) Genomewide analysis reveals characteristics of off-target sites bound by the Cas9 endonuclease. Nature Biotechnol. 32:677-683

Li Z, Liu ZB, Xing A, Moon BP, Koellhoffer JP, Juang L, Ward RT, Clifton E, Falco SC, Cigan AM. (2015) Cas9-guide RNA directed genome editing in soybean. Plant Physiology 169: 960-970

Lowder L, Malzahn A, Qi Y. (2016) Rapid Evolution of Manifold CRISPR Systems for Plant Genome Editing. Front. Plant Sci. 7:1683. doi: 10.3389/fpls.2016.01683

$\mathrm{Lu} \mathrm{Y,} \mathrm{Zhu} \mathrm{JK.} \mathrm{(2016)} \mathrm{Precise} \mathrm{editing} \mathrm{of} \mathrm{a} \mathrm{target} \mathrm{base} \mathrm{in} \mathrm{the} \mathrm{rice}$ genome using a modified CRISPR/Cas9. Molecular Plant 10: 523-525

Luo M, Wu X, Morbitzer R, Lahaye T, Ayliffe M. (2017) Genome Editing in Cereals. Proc. of the Plant and Animal Genome Conference (PAG XXV). San Diego, CA, USA. W264
Ma Y, Zhang X, Shen B, Lu Y, Chen W, Ma J, Bai L, Huang X, Zhang L. (2014) Generating rats with conditional alleles using CRISPR/Cas9. Cell Research 24:122-125. doi:10.1038/cr.2013.157

Mali P, Yang L, Esvelt KM, Aach J, Guell M, Dicarlo JE, Norville JE, Church GM. (2013) RNA-guided human genome engineering via Cas9. Science 339,823-826

Mashiko D, Young SA, Muto M, Kato H, Nozawa K, Ogawa M, Noda T, Kim YJ, Satouh Y, Fujihara Y, Ikawa M. (2014) Feasibility for a large scale mouse mutagenesis by injecting CRISPR/Cas plasmid into zygotes. Dev Growth Differ. 56(1): 122-129 doi: 10.1111/dgd.12113

Nakashima K, Ito Y, Yamaguchi-Shinozaki K. (2009) Transcriptional regulatory networks in response to abiotic stresses in Arabidopsis and grasses. Plant Physiol. 149:88-95

Niu Y, Shen B, Cui Y, Chen Y, Wang J, Wang L, Kang Y, Zhao X, Si W, Li W, Xiang AP, Zhou J, Guo X, Bi Y, Si C, Hu B, Dong G, Wang H, Zhou Z, Li T, Tan T, Pu X, Wang F, Ji S, Zhou Q, Huang X, Ji W, Sha J. (2014) Generation of gene-modified cynomolgus monkey via Cas9/RNA-mediated gene targeting in one-cell embryos. Cell 156(4):836-843 doi: 10.1016/j.cell. 2014.01.027

Puchta H and Fauser F. (2014) Synthetic nucleases for genome engineering in plants: prospects for a bright future. Plant J. 78: 727-741

Rouet P, Smih F, Jasin M. (1994) Introduction of doublestrand breaks into the genome of mouse cells by expression of a rare-cutting endonuclease. Mol. Cell Biol. 14:8096-8106

Sander JD, Joung JK. (2014) CRISPR-Cas syetms for editing, regulating and targeting genomes. Nature Biotechnology 32: 347-355 doi:10.1038/nbt.2842

Shan Q, Wang Q, Li J, Zhang Y, Chen K, Liang Z, Zhang K, Liu J, Xi JJ, Qiu JL Gao C. (2013) Targeted genome modification of crop plants using a CRISPR/Cas system. Nature Biotechnology 31(8):686-688

Sternberg SH, Redding S, Jinek M, Greene EC, Doudna JA. (2014) DNA interrogation by the CRISPR RNA-guided endonuclease Cas9. Nature 507:62-67

Sun Y. (2017) CRISPR/Cas9-Mediated Gene Targeting for Crop Improvement. Proc. of the Plant and Animal Genome Conference (PAG XXV). San Diego, CA, USA. W731

Svitashev S, Young JK, Schwartz C, Gao H, Falco SC, Cigan AM. (2015) Targeted mutagenesis, precise gene editing, and site-specific gene insertion in maize using Cas9 and Guide RNA. Plant Physiology 169:931-945

Voytas DF, Gao C. (2014) Precision genome engineering and agriculture: opportunities and regulatory challenges. PLoS Biol. 12:e1001877. doi: 10.1371/journal.pbio.1001877

Wang Y, Cheng X, Shan Q, Zhang Y, Liu J, Gao C, Qiu JL. (2014) Simultaneous editing of three homoeoalleles in hexaploid bread wheat confers heritable resistance to powdery mildew. Nature Biotechnology doi:10.1038/nbt.2969

Wang S, Zhang S, Wang W, Xiong X, Meng F, Cui X. (2015) Efficient targeted mjtageneis in potato by the CRISPR/Cas9 system Plant Cell Rep 34:1473-1476 DOI 10.1007/s00299015-1816-7 
Wang F, Wang C, Liu P, Lei C, Hao W, Gao Y, Liu YG, Zhao K. (2016) Enhanced rice blast resistance by CRISPR/Cas9-targeted mutagenesis of the ERF transcription factpr gene OsERF922. PLOS One 11 (4): e0154027. doi:10.1371/journal.pone.0154027

Wolfe SA, Nekludova L, Pabo CO. (2000) DNA Recognition by Cys2His2Zinc finger proteins. Annu. Rev, Biphys. Biomol. Struct. 3:183-212

Yang D, Xu J, Zhu T, Fan J, Lai L, Zhang J, Chen YE. (2014) Effective gene targeting in rabbits using RNA-guided Cas9 nucleases. J Mol Cell Biol. 6(1):97-99

Zhang H, Zhang J, Wei P, Zhang B, Gou F, Feng Z, Mao Y, Yang L, Zhang H, Xu N, Zhu JK. (2014) The CRISPR/Cas9 system produces specific and homozygous targeted gene editing in rice in one generation. Plant Biotechnology Journal 12:797-807

Zhu J, Song N, Sun S, Yang W, Zhao H, Song W, Lai J. (2015) Efficiency and inheritance of targeted mutagenesis in maize using CRISPR-Cas9. Journal of Genetics and Genomics 43: 25-36 